\title{
Josefilaria mackerrasae gen. et sp. nov. \\ (Nematoda : Filarioidea) \\ parasite de la Chauve-souris \\ Macroderma gigas Dobson
}

par D.-E. MOORHOUSE *, O. BAIN ** et G. WOLF *

* Department of Parasitology, University of Queensland, St-Lucia, Queensland, Australia, 4067.

* Laboratoire de Zoologie (Vers), associé au C.N.R.S., Muséum national d'Histoire naturelle, 43, rue Cuvier, F 75231 Paris Cedex 05.

\section{Résumé.}

Description de Josefilaria mackerrasae n. gen., n. sp., Filaire parasite de la chauvesouris Macroderma gigas, en Australie. Cette Filaire présente des affinités avec le genre Litomosa, mais s'en éloigne par le mélange de caractères évolués (capsule buccale réduite à un anneau pré-œsophagien; papilles labiales externes absentes) liés à des caractères primitifs ("ailes caudales » chez le $\delta$, œsophage long et divisé, vulve peu éloignée de l'anneau nerveux, nombreuses pointes caudales chez la $\%$ ).

\section{Summiary.}

Josefilaria mackerrasae gen. et sp. nov. (Nematoda : Filarioidea) a parasite of the ghost bat Macroderma gigas Dobson.

Josefilaria mackerrasae n.gen., n.sp., a filarial of the ghost bat Macroderma gigas in Australia is described. This filarioid has affinities with the genus Litomosa, but it can be separated by a variety of both more highly evolved characters (buccal capsule reduced to a pre-œsophageal ring ; external lateral papillae absent) and primitive characters ("caudal alae " of the male, long and divided œsophagus, vulva not far from the nerve ring, numerous caudal mucrons of the female).

Accepté le 5 novembre 1979. 


\section{Introduction}

En étudiant l'histopathologie de la Tique Argas macrodermae, se gorgeant sur la Chauve-souris Macroderma gigas (1) Dobson (Hoogstraal et coll., 1977), des microfilaires de petite taille d'une Filaire inconnue ont été trouvées dans le derme superficiel. La dissection des chauve-souris a permis de récolter les adultes, logés sous la peau, au-dessus des muscles pectoraux.

\section{L'hôte.}

L'hôte, $M$. gigas, de la famille des Megadermatidae, est le seul membre de ce genre (2). Actuellement sa distribution est limitée au nord et à l'ouest de l'Australie, mais elle s'étendait autrefois sur une plus grande partie du continent.

$M$. gigas niche dans les parties les plus élevées des crevasses rocheuses, en petites colonies comprenant jusqu'à 50 individus. La composition des colonies et leur emplacement changent au cours de l'année. Les chauve-souris sont aisément troublées et délaissent alors leur gîte. Elles se nourrissent d'insectes, de petits mammifères, lézards, grenouilles et même de petits oiseaux (Ride, 1970).

\section{Origine géographique.}

Johannsen's Caves, Ridgeland, Derryvale et Yaamba près de Rockhampton ; Cape Hillsborough, près de Mackay; Coen, dans le Queensland. Pine Creek, près de Catherine ; Mount Borradaile, près de Cooper's Creek et Nourlangie Rock, Arnhemland dans le Northern Territory, Australie.

\section{Localisation.}

Les Filaires sont logées dans des dépressions sur les faisceaux des muscles pectoraux, ou sont partiellement encapsulées dans le derme profond qui recouvre ces muscles et alors enveloppées d'une fine membrane formée de fibroblastes, lymphocytes, plasmocytes, polynucléaires et cellules géantes.

Sur 26 chauve-souris, 10 ont des vers intacts et 2 des vers dégénérés, ou partiellement calcifiés.

Sur coupes, de nombreuses microfilaires s'observent dans la peau des ailes, au niveau du derme superficiel. La plupart sont situées entre les fibres de collagène ; quelques-unes sont à l'intérieur des petits vaisseaux lymphatiques, mais aucune dans les vaisseaux sanguins. Il n'y a également aucune microfilaire dans des gouttes épaisses colorées au Giemsa, provenant de sang prélevé dans les artères médianes de 8 chau-

(1) « Ghost " ou « false vampire bat ».

(2) 2 autres genres existent en Asie et en Afrique (Walker, 1968). 
ve-souris. Les coupes histologiques montrent que les microfilaires ont tendance à s'accumuler au voisinage des piqûres des arthropodes, ainsi que l'a remarqué Moorhouse (1969) avec des microfilaires dermiques d'une Filaire inconnue, chez le Marsupial volant Schoinobates volans.

\section{Matériel.}

10 \& et 16 ô. Holotype $\%$ (Ref. Q.M. 11608) et allotype ô (Réf. Q.M. 11609) trouvés in copula, provenant de Ridgeland, près de Rockhampton, Queensland, déposés au Queensland Museum, Brisbane, Australie. Autres spécimens déposés au Museum national d'Histoire naturelle, Paris ; South Australian Museum, Adelaide ; U.S. National Museum Helminthological Collection, Beltsville Parasitological Laboratory, Beltsville, Maryland, U.S.A. et Dept. of Parasitology, University of Queensland, St-Lucia, Queensland, Australie.

\section{Description.} cimens.

— Femelle (fig. 1 A à E) : 7 spécimens entiers et des fragments de 3 autres spé-

Corps avec stries transversales régulièrement espacées, absentes aux extrémités du ver (fig. $2 \mathrm{C}$ ), 2 petits épaississements longitudinaux latéraux (fig. $2 \mathrm{E}$ ). 4 papilles sur la tête, plus les phasmides (fig. $1 \mathrm{E}$ ) ; bouche ronde ; présence d'un anneau cuticulaire pré-œsophagien. Esophage très long, clairement divisé en portions glandulaire et musculaire, celle-ci faisant approximativement le dixième de la longueur totale de l'œsophage. Anneau nerveux à mi-hauteur de l'œsophage musculaire. Vulve antérieure, étirée en fente. Queue ornée à son extrémité par plusieurs petites pointes cuticulaires; phasmides subterminales.

\section{Dimensions.}

o holotype : corps long de $78-20 \mathrm{~mm}$, large de $270 \mu \mathrm{m}$ au niveau de la vulve et, au maximum, de $350 \mu \mathrm{m}$; anneau nerveux à $140 \mu \mathrm{m}$ de la tête ; anneau pré-œsophagien haut de $3 \mu \mathrm{m}$; œsophage long de $2210 \mu \mathrm{m}$ (portion musculaire longue de $240 \mu \mathrm{m}$ ) ; vulve à $370 \mu \mathrm{m}$ de l'apex; queue longue de $280 \mu \mathrm{m}$.

Mensurations extrêmes des 6 autres entières : corps long de 50,00 à $88,27 \mathrm{~mm}$; largeur à la vulve de 270 à $330 \mu \mathrm{m}$; largeur maximum de 270 à $350 \mu \mathrm{m}$; œsophage long de 1940 à $2400 \mu \mathrm{m}$; vulve de 330 à $460 \mu \mathrm{m}$ de la tête ; queue longue de 190 à $380 \mu \mathrm{m}$.

Mâle (fig. $1 F$ à $O$ ) : 15 spécimens entiers et des fragments d'un autre ô. Mêmes stries cuticulaires que chez la $\uparrow$, mais s'étendant jusqu'à l'extrémité de la queue et présentant un relief irrégulier dans la région du cloaque (fig. $2 \mathrm{D}$ ), donnant l'apparence de rugosités en microscopie optique. Deux petits épaississements longitudinaux latéraux. CEsophage divisé comme chez la $\uparrow$, la portion musculaire faisant le 1/7 de 

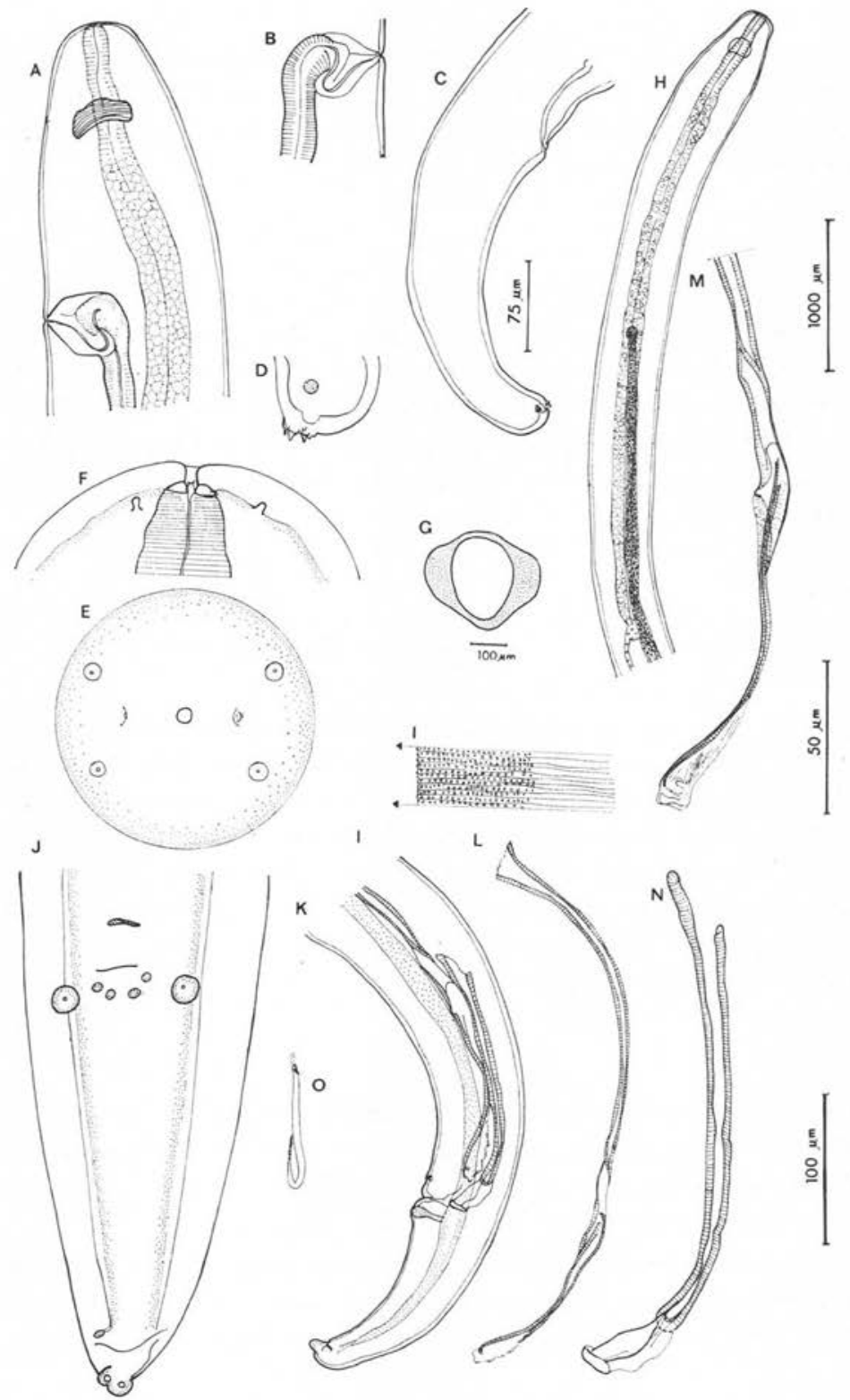

ᄂ
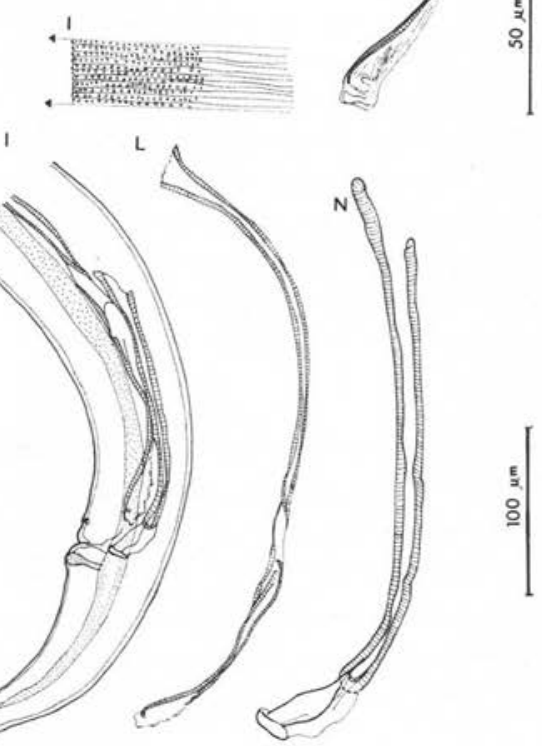

Fig. 1. Josefilaria mackerrasae n. gen., n.sp.; A à E, $q$; A : région antérieure, vue latérale; B : détail du sphincter et de la vulve; C : queue, vue latérale; D : extrémité caudale, vue latérale; $\mathrm{E}$ : tête, vue apicale; $\mathrm{F}$ à $\mathrm{N}:{ }^{0}$ allotype ; $\mathrm{F}$ : tête, vue latérale; $\mathrm{G}$ : coupe transversale de la queue montrant les « ailes latérales"; $\mathrm{H}$ : région antérieure, vue latérale; I : area rugosa, vue latérale ; J : queue, vue ventrale ; $\mathrm{K}$ : région caudale, vue latérale; $\mathrm{L}$ : spicule gauche, vue latérale; $\mathrm{M}$ : idem, lame; $\mathrm{N}$ : spicule droit, vue latérale ; $\mathrm{O}$ : microfilaire mûre, extraite de la $\$$. 

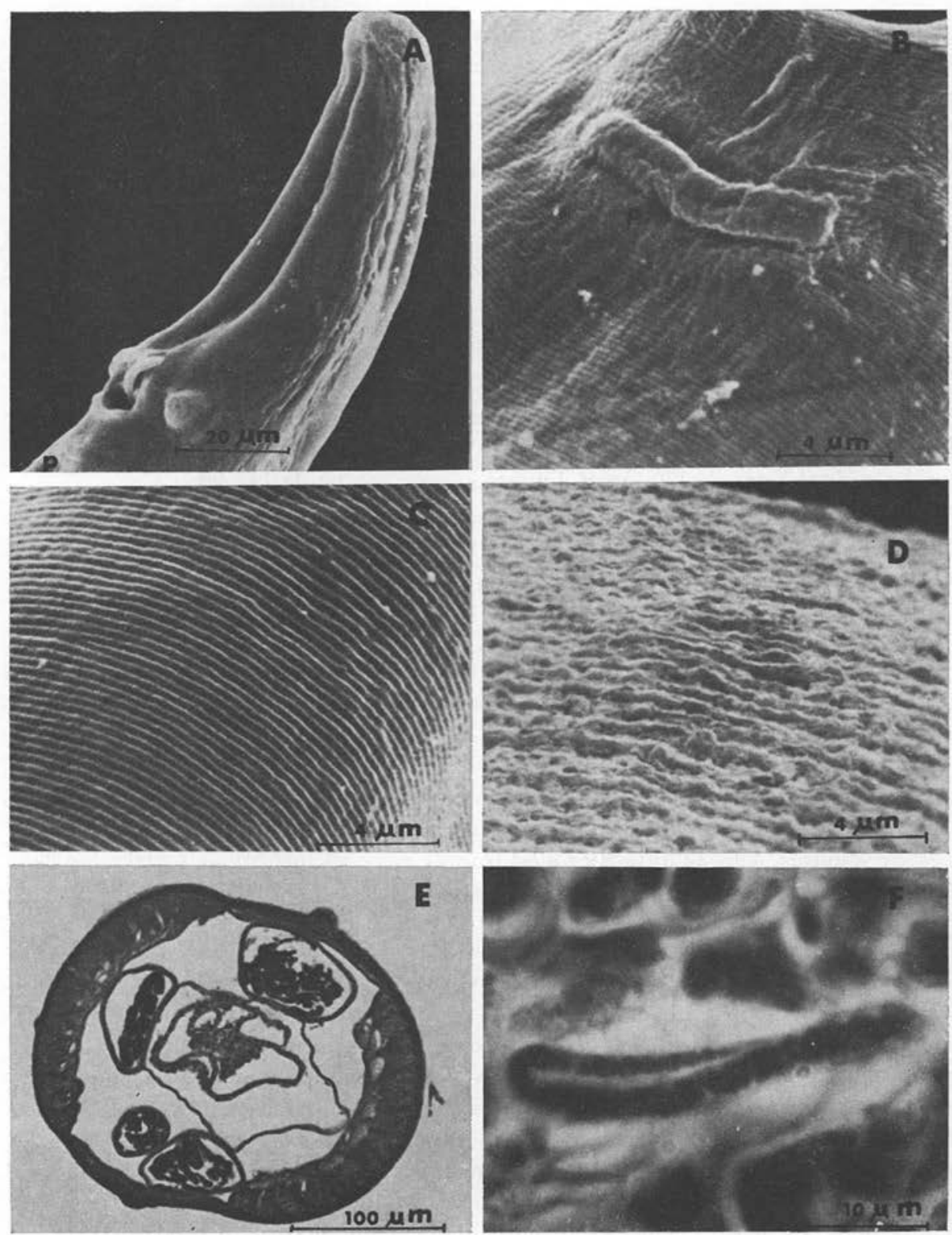

Fig. 2. Josefilaria mackerrasae n. gen., n. sp.; A : queue du $\delta$ au microscope à balayage $(\mathrm{MB}) ; \mathrm{p}=$ papille impaire précloacale ; $\mathrm{B}$ : détail de la papille $(\mathrm{MB}) ; \mathrm{C}: \stackrel{+}{\text {, stries cuti- }}$ culaires transversales $(\mathrm{MB}) ; \mathrm{D}: \delta^{*}$, stries tranversales dans la région cloacale $(\mathrm{MB})$; $\mathrm{E}$ : renflements cuticulaires longitudinaux (coupe transversale d'1 \&); F : microfilaire dans le derme superficiel (coloration de Gomori). 
la longueur totale de l'œsophage. Région postérieure enroulée sur 4 spires. Cuticule des faces latérales très épaissies un peu en avant du cloaque jusqu'à l'extrémité de la queue (fig. $1 \mathrm{G}$ et $\mathrm{J}$ ). Area rugosa formée de rangées transversales de petites perles cuticulaires s'étendant de 2700 à $6600 \mu \mathrm{m}$ de l'extrémité caudale (fig. $1 \mathrm{I}$ ).

Disposition des papilles caudales représentées sur la fig. $1 \mathrm{~J}$; extrémité caudale courbée vers la face ventrale, légèrement bilobée, avec 2 corpuscules très réfringents (phasmides ?).

Spicule gauche avec lame complexe, membraneuse à son extrémité ; spicule droit puissant, à extrémité en cuiller (fig. $1 \mathrm{~K}, L, M, N$ ).

Dimensions.

of allotype : corps long de $37,20 \mathrm{~mm}$, large de $240 \mu \mathrm{m}$; anneau nerveux à $130 \mu \mathrm{m}$ de l'apex ; œsophage long de $2100 \mu \mathrm{m}$, et portion musculaire de $320 \mu \mathrm{m}$; spicules gauche et droit longs de $390 \mu \mathrm{m}$ et $150 \mu \mathrm{m}$; queue longue de $145 \mu \mathrm{m}$.

Dimensions extrêmes des autres ô : corps long de 27,00 à $46,60 \mathrm{~mm}$, large de 210 à $260 \mu \mathrm{m}$; œsophage long de $1410 \mu \mathrm{m}$ à $2130 \mu \mathrm{m}$; spicule gauche long de $310 \mu \mathrm{m}$ à $460 \mu \mathrm{m}$; spicule droit long de 150 à $200 \mu \mathrm{m}$; queue longue de 100 à $140 \mu \mathrm{m}$.

- Microfilaires (fig. $1 \mathrm{O}$ et $2 \mathrm{~F}$ ).

Microfilaires mûres pliées dans une gaine courte, dans l'ovéjecteur ; avec ou sans gaine dans la peau. Elles mesurent 51 à $76 \mu \mathrm{m}$ de longueur, avec une moyenne de $59 \mu \mathrm{m}$ (12 spécimens) et sont larges de 2,5 à $3 \mu \mathrm{m}$. Anneau nerveux à $13-19 \mu \mathrm{m}$ de l'extrémité antérieure, avec une moyenne de 15,5 $\mathrm{m}$. Extrémité antérieure amincie et obtuse, queue effilée. Extrémité antérieure de la colonne nucléaire pyramidale ; noyaux s'étendant presque jusqu'au bout de la queue, les 2 derniers noyaux étant remarquablement allongés. Aucune différence n'est trouvée entre les microfilaires prélevées dans l'utérus et celles observées sur les coupes de peau.

\section{Discussion}

Il existe chez cette Filaire des caractères qui évoquent les Litomosa Yorke et Maplestone, 1927 : petit nombre de papilles caudales groupées près du cloaque, pointes caudales chez la + , microfilaires mûres repliées dans une gaine courte, souvent libres dans le derme (cf. Petit, sous presse (1). Ce matériel présente, par ailleurs, des caractères bien particuliers, qui ne permettent pas de le placer dans ce genre : présence d'épais renflements cuticulaires latéraux sur la queue du $\hat{\delta}$, œsophage non atrophié ( $2 \mathrm{~mm}$, au lieu d'1 $\mathrm{mm}$ au maximum chez les Litomosa), vulve peu éloignée de

(1) Chez L. hugoti Petit, sous presse : "dans l'ovéjecteur et l'utérus, les microfilaires sont pliées en deux à l'intérieur d'une gaine en forme d'œuf allongé... Dans une bıopsie exsangue faite à l'oreille, les microfilaires sont nombreuses et sont dégagées de leur gaine ». 
l'anneau nerveux, et surtout existence d'un simple anneau cuticulaire pré-œsophagien au lieu d'une capsule buccale segmentée.

Il s'agit donc d'un genre nouveau, que nous dédions au $\mathrm{D}^{\mathrm{r}} \mathrm{M}$. Josephine Mackerras, en reconnaissance de sa large contribution à la connaissance de la faune parasite d'Australie; nous le nommons Josefilaria mackerrasae n. gen., n. sp., et proposons pour ce genre la définition suivante :

Josefilaria n. gen: Onchocercidae, Onchocercinae, capsule buccale réduite à un anneau pré-œsophagien; œsophage long et divisé; queue longue munie de pointes terminales chez la + et de renflements cuticulaires latéraux chez le ô ; area rugosa présente; papilles caudales peu nombreuses, groupées près du cloaque; microfilaire mûre, petite et repliée dans la gaine ; parasites de chauve-souris.

Espèce type : Josefilaria mackerrasae n. sp., parasite de Macroderma gigas, en Australie.

Le genre Litomosa ne diffère pas très profondément des Dipetalonema sensu largo et peut même être considéré comme une branche primitive de cette lignée (Chabaud et Bain, 1976).

Nous interprétons Josefilaria mackerrasae n. gen., n. sp., comme une forme relique du groupe Litomosa et, comme il est fréquent dans ces formes reliques, nous trouvons un mélange de caractères primitifs (queue du ồ à "ailes caudales ", spicules puissants et de forme compliquée, œsophage grand, avec division musculoglandulaire bien marquée, vulve bien antérieure, extrémité caudale de la $q$ ornée de plusieurs pointes, microfilaires dermiques) et de caractères évolués (capsule buccale non segmentée, réduite à un anneau comme chez certains Dipetalonema; papilles labiales externes absentes).

\section{REMERCIEMENTS}

Nous désirons remercier M. Ian Humphery-Smith, le $\mathrm{D}^{\mathrm{r}}$ John F. Nelson, M. Steve Van Dyke, les $\mathrm{D}^{\mathrm{rs}} \mathrm{J}$ John Calaby et D. M. Spratt, qui, soit nous ont autorisé à disséquer les chauvesouris qu'ils avaient en collection, soit ont récolté les vers pour nous.

Nous remercions également M. John Hardy pour son aide au microscope à balayage.

\section{Bibliographie}

Chabaud A.-G., Bain O. (1976): La lignée Depitalonema. Nouvel essai de classification. Ann. Parasitol. Hum. Comp., 51, 365-397.

Hoogstraal H., Moorhouse D.E., Wolf G., Wassef H. Y. (1977) : Bat ticks of the genus Argas (Ixodoidea: Argasidae). A. (Carios) macrodermae New species from Queensland, Australia. Ann. Ent. Soc. America, 70, 861-870.

Moorhouse D.E. (1969: The accumulation of microfilariae beneath the sites of attachment of Ixodes tasmani. Trans. R. Soc. Trop. Med. Hyg., 63, 22.

Petit G.: Sur les Filaires du genre Litomosa, parasites de Chauve-souris. Bull. Mus. Nat. Hist. Nat.

Ride W. D. L. (1970) : A guide to the native nammals of Australia. Melbourne. Oxford University Press, London, Wellington, New-York, 249 p.

Walker E. P. (1968) : Mammals of the world. Vol. 1, 2 édition, Johns Hopkins Press, Baltimore, pp. 248-251. 